Supplementary Information

\title{
Gas Phase Protein Folding Triggered by Proton Stripping Generates Inside-Out Structures: A Molecular Dynamics Simulation Study
}

\author{
Alexander I. M. Sever and Lars Konermann * \\ Department of Chemistry, The University of Western Ontario, London, Ontario, \\ N6A 5B7, Canada
}

This file contains:

Table S1: Charge patterns for static-proton runs.

Figure S1: Overlay of different $3+$ gas phase structures.

Figure S2: $R_{g}$ values generated without temperature annealing. 


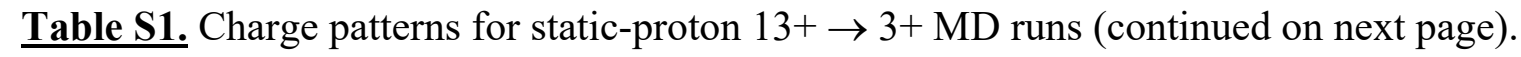

Proton Removal from Acidic Side Chains, Condition \#1

\begin{tabular}{|c|c|c|c|c|c|c|c|c|c|c|c|c|c|c|c|c|c|c|c|c|c|c|c|c|c|}
\hline & $\mathrm{NT}$ & $\mathrm{K}$ & $\mathrm{K}$ & $\mathrm{E}$ & $\mathrm{E}$ & $\mathrm{D}$ & $\mathrm{E}$ & $\mathrm{K}$ & $\mathrm{K}$ & $\mathrm{D}$ & $\mathrm{K}$ & $\mathrm{E}$ & $\mathrm{D}$ & $\mathrm{R}$ & $\mathrm{K}$ & $\mathrm{E}$ & $\mathrm{D}$ & $\mathrm{R}$ & $\mathrm{D}$ & $\mathrm{K}$ & $\mathrm{E}$ & $\mathrm{H}$ & $\mathrm{R}$ & $\mathrm{R}$ & $\mathrm{CT}$ \\
\hline+13 & + & + & + & 0 & 0 & 0 & 0 & + & + & 0 & + & 0 & 0 & + & + & 0 & 0 & + & 0 & + & 0 & + & + & + & 0 \\
\hline+12 & + & + & + & 0 & 0 & 0 & 0 & + & + & 0 & + & 0 & - & + & + & 0 & 0 & + & 0 & + & 0 & + & + & + & 0 \\
\hline+11 & + & + & + & 0 & 0 & - & 0 & + & + & 0 & + & 0 & - & + & + & 0 & 0 & + & 0 & + & 0 & + & + & + & 0 \\
\hline+10 & + & + & + & 0 & 0 & - & 0 & + & + & 0 & + & 0 & - & + & + & 0 & 0 & + & - & + & 0 & + & + & + & 0 \\
\hline+9 & + & + & + & 0 & 0 & - & 0 & + & + & 0 & + & 0 & - & + & + & 0 & - & + & - & + & 0 & + & + & + & 0 \\
\hline+8 & + & + & + & 0 & 0 & - & 0 & + & + & - & + & 0 & - & + & + & 0 & - & + & - & + & 0 & + & + & + & 0 \\
\hline+7 & + & + & + & 0 & 0 & - & - & + & + & - & + & 0 & - & + & + & 0 & - & + & - & + & 0 & + & + & + & 0 \\
\hline+6 & + & + & + & - & 0 & - & - & + & + & - & + & 0 & - & + & + & 0 & - & + & - & + & 0 & + & + & + & 0 \\
\hline+5 & + & + & + & - & 0 & - & - & + & + & - & + & 0 & - & + & + & 0 & - & + & - & + & - & + & + & + & 0 \\
\hline+4 & + & + & + & - & - & - & - & + & + & - & + & 0 & - & + & + & 0 & - & + & - & + & - & + & + & + & 0 \\
\hline+3 & + & + & + & - & - & - & - & + & + & - & + & 0 & - & + & + & - & - & + & - & + & - & + & + & + & 0 \\
\hline
\end{tabular}

Proton Removal from Acidic Side Chains, Condition \#2

\begin{tabular}{|c|c|c|c|c|c|c|c|c|c|c|c|c|c|c|c|c|c|c|c|c|c|c|c|c|c|}
\hline & $\mathrm{NT}$ & $\mathrm{K}$ & $\mathrm{K}$ & $\mathrm{E}$ & $\mathrm{E}$ & $\mathrm{D}$ & $\mathrm{E}$ & $\mathrm{K}$ & $\mathrm{K}$ & $\mathrm{D}$ & $\mathrm{K}$ & $\mathrm{E}$ & $\mathrm{D}$ & $\mathrm{R}$ & $\mathrm{K}$ & $\mathrm{E}$ & $\mathrm{D}$ & $\mathrm{R}$ & $\mathrm{D}$ & $\mathrm{K}$ & $\mathrm{E}$ & $\mathrm{H}$ & $\mathrm{R}$ & $\mathrm{R}$ & $\mathrm{CT}$ \\
\hline+13 & + & + & + & 0 & 0 & 0 & 0 & + & + & 0 & + & 0 & 0 & + & + & 0 & 0 & + & 0 & + & 0 & + & + & + & 0 \\
\hline+12 & + & + & + & 0 & 0 & - & 0 & + & + & 0 & + & 0 & 0 & + & + & 0 & 0 & + & 0 & + & 0 & + & + & + & 0 \\
\hline+11 & + & + & + & 0 & 0 & - & 0 & + & + & - & + & 0 & 0 & + & + & 0 & 0 & + & 0 & + & 0 & + & + & + & 0 \\
\hline+10 & + & + & + & 0 & 0 & - & 0 & + & + & - & + & 0 & - & + & + & 0 & 0 & + & 0 & + & 0 & + & + & + & 0 \\
\hline+9 & + & + & + & 0 & 0 & - & 0 & + & + & - & + & 0 & - & + & + & 0 & - & + & 0 & + & 0 & + & + & + & 0 \\
\hline+8 & + & + & + & 0 & 0 & - & 0 & + & + & - & + & 0 & - & + & + & 0 & - & + & - & + & 0 & + & + & + & 0 \\
\hline+7 & + & + & + & - & 0 & - & 0 & + & + & - & + & 0 & - & + & + & 0 & - & + & - & + & 0 & + & + & + & 0 \\
\hline+6 & + & + & + & - & - & - & 0 & + & + & - & + & 0 & - & + & + & 0 & - & + & - & + & 0 & + & + & + & 0 \\
\hline+5 & + & + & + & - & - & - & - & + & + & - & + & 0 & - & + & + & 0 & - & + & - & + & 0 & + & + & + & 0 \\
\hline+4 & + & + & + & - & - & - & - & + & + & - & + & - & - & + & + & 0 & - & + & - & + & 0 & + & + & + & 0 \\
\hline+3 & + & + & + & - & - & - & - & + & + & - & + & - & - & + & + & - & - & + & - & + & 0 & + & + & + & 0 \\
\hline
\end{tabular}

Proton Removal from Acidic Side Chains, Condition \#3

\begin{tabular}{|c|c|c|c|c|c|c|c|c|c|c|c|c|c|c|c|c|c|c|c|c|c|c|c|c|c|}
\hline & $\mathrm{NT}$ & $\mathrm{K}$ & $\mathrm{K}$ & $\mathrm{E}$ & $\mathrm{E}$ & $\mathrm{D}$ & $\mathrm{E}$ & $\mathrm{K}$ & $\mathrm{K}$ & $\mathrm{D}$ & $\mathrm{K}$ & $\mathrm{E}$ & $\mathrm{D}$ & $\mathrm{R}$ & $\mathrm{K}$ & $\mathrm{E}$ & $\mathrm{D}$ & $\mathrm{R}$ & $\mathrm{D}$ & $\mathrm{K}$ & $\mathrm{E}$ & $\mathrm{H}$ & $\mathrm{R}$ & $\mathrm{R}$ & $\mathrm{CT}$ \\
\hline+13 & + & + & + & 0 & 0 & 0 & 0 & + & + & 0 & + & 0 & 0 & + & + & 0 & 0 & + & 0 & + & 0 & + & + & + & 0 \\
\hline+12 & + & + & + & 0 & 0 & 0 & 0 & + & + & 0 & + & 0 & 0 & + & + & 0 & 0 & + & 0 & + & - & + & + & + & 0 \\
\hline+11 & + & + & + & 0 & 0 & - & 0 & + & + & 0 & + & 0 & 0 & + & + & 0 & 0 & + & 0 & + & - & + & + & + & 0 \\
\hline+10 & + & + & + & 0 & 0 & - & 0 & + & + & 0 & + & 0 & 0 & + & + & 0 & 0 & + & - & + & - & + & + & + & 0 \\
\hline+9 & + & + & + & 0 & 0 & - & 0 & + & + & 0 & + & 0 & 0 & + & + & 0 & - & + & - & + & - & + & + & + & 0 \\
\hline+8 & + & + & + & 0 & - & - & 0 & + & + & 0 & + & 0 & 0 & + & + & 0 & - & + & - & + & - & + & + & + & 0 \\
\hline+7 & + & + & + & 0 & - & - & 0 & + & + & - & + & 0 & 0 & + & + & 0 & - & + & - & + & - & + & + & + & 0 \\
\hline+6 & + & + & + & 0 & - & - & 0 & + & + & - & + & - & 0 & + & + & 0 & - & + & - & + & - & + & + & + & 0 \\
\hline+5 & + & + & + & 0 & - & - & 0 & + & + & - & + & - & - & + & + & 0 & - & + & - & + & - & + & + & + & 0 \\
\hline+4 & + & + & + & - & - & - & 0 & + & + & - & + & - & - & + & + & 0 & - & + & - & + & - & + & + & + & 0 \\
\hline+3 & + & + & + & - & - & - & 0 & + & + & - & + & - & - & + & + & - & - & + & - & + & - & + & + & + & 0 \\
\hline
\end{tabular}


Proton Removal from Basic Moieties, Condition \#1

\begin{tabular}{|c|c|c|c|c|c|c|c|c|c|c|c|c|c|c|c|c|c|c|c|c|c|c|c|c|c|}
\hline & $\mathrm{NT}$ & $\mathrm{K}$ & $\mathrm{K}$ & $\mathrm{E}$ & $\mathrm{E}$ & $\mathrm{D}$ & $\mathrm{E}$ & $\mathrm{K}$ & $\mathrm{K}$ & $\mathrm{D}$ & $\mathrm{K}$ & $\mathrm{E}$ & $\mathrm{D}$ & $\mathrm{R}$ & $\mathrm{K}$ & $\mathrm{E}$ & $\mathrm{D}$ & $\mathrm{R}$ & $\mathrm{D}$ & $\mathrm{K}$ & $\mathrm{E}$ & $\mathrm{H}$ & $\mathrm{R}$ & $\mathrm{R}$ & $\mathrm{CT}$ \\
\hline+13 & + & + & + & 0 & 0 & 0 & 0 & + & + & 0 & + & 0 & 0 & + & + & 0 & 0 & + & 0 & + & 0 & + & + & + & 0 \\
\hline+12 & + & + & + & 0 & 0 & 0 & 0 & + & + & 0 & + & 0 & 0 & + & + & 0 & 0 & + & 0 & + & 0 & 0 & + & + & 0 \\
\hline+11 & 0 & + & + & 0 & 0 & 0 & 0 & + & + & 0 & + & 0 & 0 & + & + & 0 & 0 & + & 0 & + & 0 & 0 & + & + & 0 \\
\hline+10 & 0 & 0 & + & 0 & 0 & 0 & 0 & + & + & 0 & + & 0 & 0 & + & + & 0 & 0 & + & 0 & + & 0 & 0 & + & + & 0 \\
\hline+9 & 0 & 0 & 0 & 0 & 0 & 0 & 0 & + & + & 0 & + & 0 & 0 & + & + & 0 & 0 & + & 0 & + & 0 & 0 & + & + & 0 \\
\hline+8 & 0 & 0 & 0 & 0 & 0 & 0 & 0 & 0 & + & 0 & + & 0 & 0 & + & + & 0 & 0 & + & 0 & + & 0 & 0 & + & + & 0 \\
\hline+7 & 0 & 0 & 0 & 0 & 0 & 0 & 0 & 0 & 0 & 0 & + & 0 & 0 & + & + & 0 & 0 & + & 0 & + & 0 & 0 & + & + & 0 \\
\hline+6 & 0 & 0 & 0 & 0 & 0 & 0 & 0 & 0 & 0 & 0 & 0 & 0 & 0 & + & + & 0 & 0 & + & 0 & + & 0 & 0 & + & + & 0 \\
\hline+5 & 0 & 0 & 0 & 0 & 0 & 0 & 0 & 0 & 0 & 0 & 0 & 0 & 0 & + & 0 & 0 & 0 & + & 0 & + & 0 & 0 & + & + & 0 \\
\hline+4 & 0 & 0 & 0 & 0 & 0 & 0 & 0 & 0 & 0 & 0 & 0 & 0 & 0 & + & 0 & 0 & 0 & + & 0 & 0 & 0 & 0 & + & + & 0 \\
\hline+3 & 0 & 0 & 0 & 0 & 0 & 0 & 0 & 0 & 0 & 0 & 0 & 0 & 0 & 0 & 0 & 0 & 0 & + & 0 & 0 & 0 & 0 & + & + & 0 \\
\hline
\end{tabular}

Proton Removal from Basic Moieties, Condition \#2

\begin{tabular}{|c|c|c|c|c|c|c|c|c|c|c|c|c|c|c|c|c|c|c|c|c|c|c|c|c|c|}
\hline & $\mathrm{NT}$ & $\mathrm{K}$ & $\mathrm{K}$ & $\mathrm{E}$ & $\mathrm{E}$ & $\mathrm{D}$ & $\mathrm{E}$ & $\mathrm{K}$ & $\mathrm{K}$ & $\mathrm{D}$ & $\mathrm{K}$ & $\mathrm{E}$ & $\mathrm{D}$ & $\mathrm{R}$ & $\mathrm{K}$ & $\mathrm{E}$ & $\mathrm{D}$ & $\mathrm{R}$ & $\mathrm{D}$ & $\mathrm{K}$ & $\mathrm{E}$ & $\mathrm{H}$ & $\mathrm{R}$ & $\mathrm{R}$ & $\mathrm{CT}$ \\
\hline+13 & + & + & + & 0 & 0 & 0 & 0 & + & + & 0 & + & 0 & 0 & + & + & 0 & 0 & + & 0 & + & 0 & + & + & + & 0 \\
\hline+12 & + & + & + & 0 & 0 & 0 & 0 & + & + & 0 & + & 0 & 0 & + & + & 0 & 0 & + & 0 & + & 0 & 0 & + & + & 0 \\
\hline+11 & + & + & + & 0 & 0 & 0 & 0 & + & + & 0 & + & 0 & 0 & + & + & 0 & 0 & + & 0 & 0 & 0 & 0 & + & + & 0 \\
\hline+10 & + & + & + & 0 & 0 & 0 & 0 & + & + & 0 & + & 0 & 0 & + & 0 & 0 & 0 & + & 0 & 0 & 0 & 0 & + & + & 0 \\
\hline+9 & + & + & + & 0 & 0 & 0 & 0 & + & + & 0 & 0 & 0 & 0 & + & 0 & 0 & 0 & + & 0 & 0 & 0 & 0 & + & + & 0 \\
\hline+8 & + & + & + & 0 & 0 & 0 & 0 & + & 0 & 0 & 0 & 0 & 0 & + & 0 & 0 & 0 & + & 0 & 0 & 0 & 0 & + & + & 0 \\
\hline+7 & + & + & + & 0 & 0 & 0 & 0 & 0 & 0 & 0 & 0 & 0 & 0 & + & 0 & 0 & 0 & + & 0 & 0 & 0 & 0 & + & + & 0 \\
\hline+6 & + & + & 0 & 0 & 0 & 0 & 0 & 0 & 0 & 0 & 0 & 0 & 0 & + & 0 & 0 & 0 & + & 0 & 0 & 0 & 0 & + & + & 0 \\
\hline+5 & + & 0 & 0 & 0 & 0 & 0 & 0 & 0 & 0 & 0 & 0 & 0 & 0 & + & 0 & 0 & 0 & + & 0 & 0 & 0 & 0 & + & + & 0 \\
\hline+4 & 0 & 0 & 0 & 0 & 0 & 0 & 0 & 0 & 0 & 0 & 0 & 0 & 0 & + & 0 & 0 & 0 & + & 0 & 0 & 0 & 0 & + & + & 0 \\
\hline+3 & 0 & 0 & 0 & 0 & 0 & 0 & 0 & 0 & 0 & 0 & 0 & 0 & 0 & + & 0 & 0 & 0 & 0 & 0 & 0 & 0 & 0 & + & + & 0 \\
\hline
\end{tabular}

Proton Removal from Basic Moieties, Condition \#3

\begin{tabular}{|c|c|c|c|c|c|c|c|c|c|c|c|c|c|c|c|c|c|c|c|c|c|c|c|c|c|}
\hline & $\mathrm{NT}$ & $\mathrm{K}$ & $\mathrm{K}$ & $\mathrm{E}$ & $\mathrm{E}$ & $\mathrm{D}$ & $\mathrm{E}$ & $\mathrm{K}$ & $\mathrm{K}$ & $\mathrm{D}$ & $\mathrm{K}$ & $\mathrm{E}$ & $\mathrm{D}$ & $\mathrm{R}$ & $\mathrm{K}$ & $\mathrm{E}$ & $\mathrm{D}$ & $\mathrm{R}$ & $\mathrm{D}$ & $\mathrm{K}$ & $\mathrm{E}$ & $\mathrm{H}$ & $\mathrm{R}$ & $\mathrm{R}$ & $\mathrm{CT}$ \\
\hline+13 & + & + & + & 0 & 0 & 0 & 0 & + & + & 0 & + & 0 & 0 & + & + & 0 & 0 & + & 0 & + & 0 & + & + & + & 0 \\
\hline+12 & + & + & + & 0 & 0 & 0 & 0 & + & + & 0 & + & 0 & 0 & + & + & 0 & 0 & + & 0 & + & 0 & 0 & + & + & 0 \\
\hline+11 & + & + & 0 & 0 & 0 & 0 & 0 & + & + & 0 & + & 0 & 0 & + & + & 0 & 0 & + & 0 & + & 0 & 0 & + & + & 0 \\
\hline+10 & + & + & 0 & 0 & 0 & 0 & 0 & + & 0 & 0 & + & 0 & 0 & + & + & 0 & 0 & + & 0 & + & 0 & 0 & + & + & 0 \\
\hline+9 & 0 & + & 0 & 0 & 0 & 0 & 0 & + & 0 & 0 & + & 0 & 0 & + & + & 0 & 0 & + & 0 & + & 0 & 0 & + & + & 0 \\
\hline+8 & 0 & + & 0 & 0 & 0 & 0 & 0 & 0 & 0 & 0 & + & 0 & 0 & + & + & 0 & 0 & + & 0 & + & 0 & 0 & + & + & 0 \\
\hline+7 & 0 & + & 0 & 0 & 0 & 0 & 0 & 0 & 0 & 0 & + & 0 & 0 & + & + & 0 & 0 & + & 0 & 0 & 0 & 0 & + & + & 0 \\
\hline+6 & 0 & + & 0 & 0 & 0 & 0 & 0 & 0 & 0 & 0 & 0 & 0 & 0 & + & + & 0 & 0 & + & 0 & 0 & 0 & 0 & + & + & 0 \\
\hline+5 & 0 & 0 & 0 & 0 & 0 & 0 & 0 & 0 & 0 & 0 & 0 & 0 & 0 & + & + & 0 & 0 & + & 0 & 0 & 0 & 0 & + & + & 0 \\
\hline+4 & 0 & 0 & 0 & 0 & 0 & 0 & 0 & 0 & 0 & 0 & 0 & 0 & 0 & + & 0 & 0 & 0 & + & 0 & 0 & 0 & 0 & + & + & 0 \\
\hline+3 & 0 & 0 & 0 & 0 & 0 & 0 & 0 & 0 & 0 & 0 & 0 & 0 & 0 & + & 0 & 0 & 0 & + & 0 & 0 & 0 & 0 & + & 0 & 0 \\
\hline
\end{tabular}




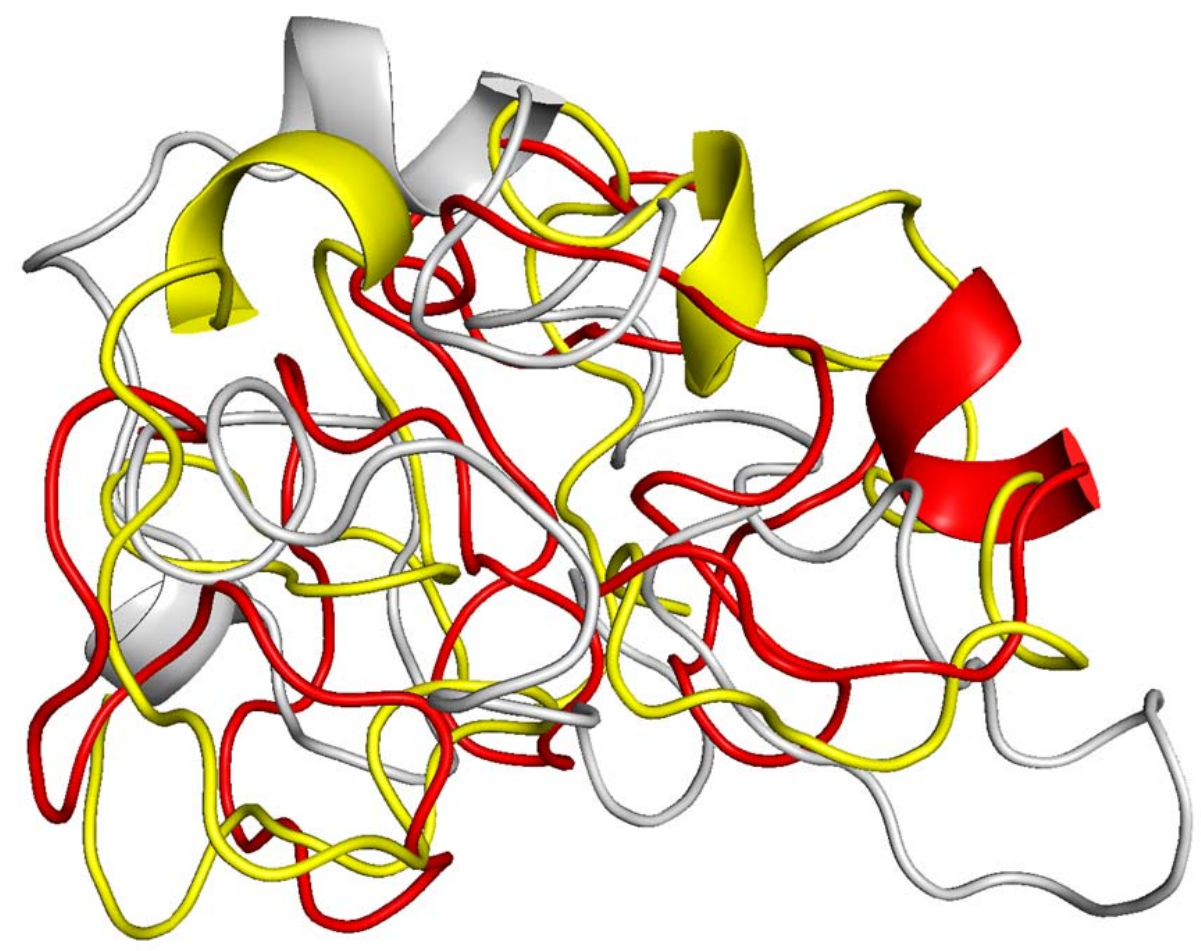

Figure S1. Overlay of ubiquitin 3+ backbone cartoons obtained in three separate mobile-proton runs after stepwise $13+\rightarrow 3+$ proton stripping with temperature annealing. The three chains were structurally aligned in Pymol. This figure reveals that folding in the gas phase does not produce a uniform final conformation. 


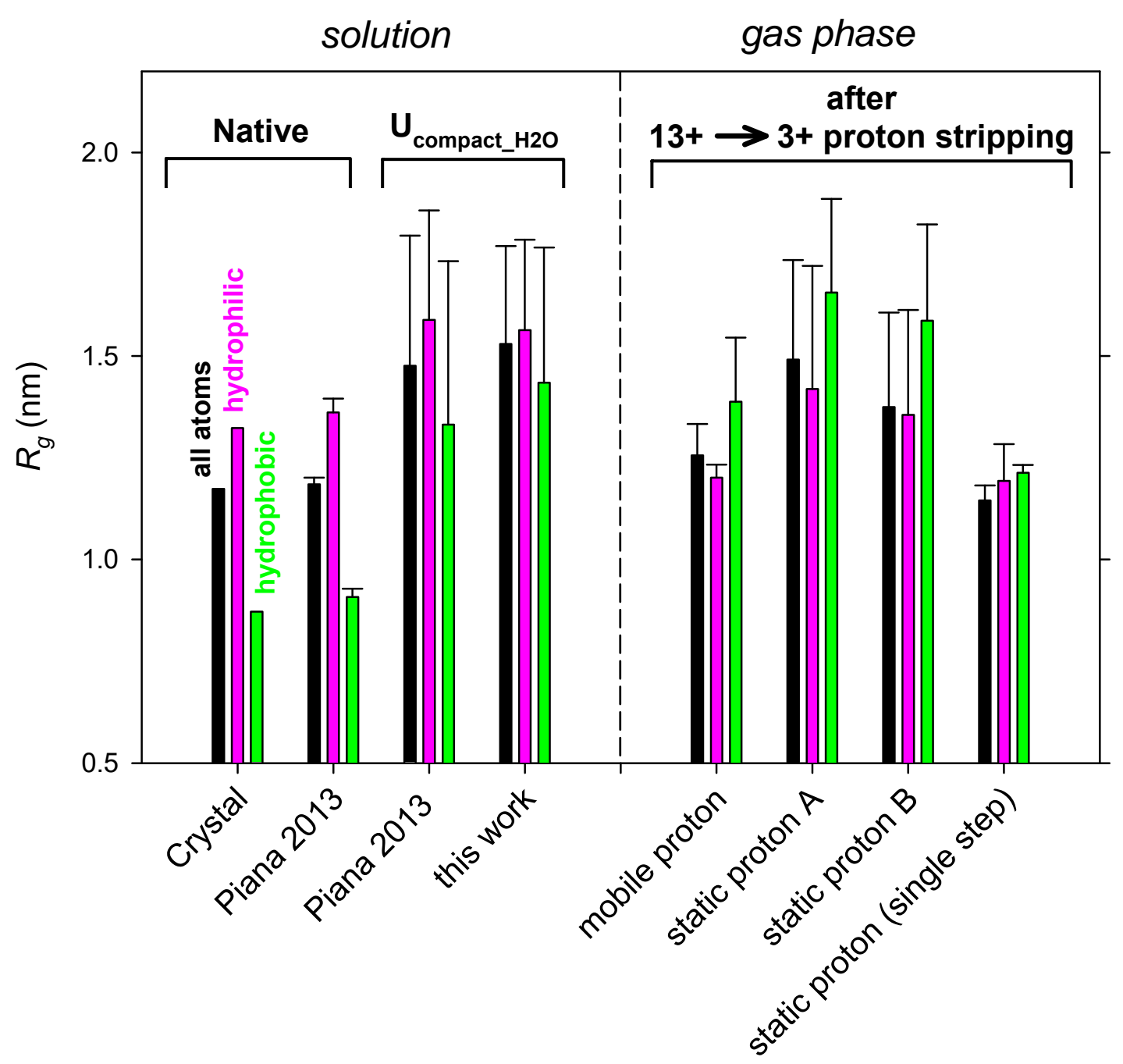

Figure S2. $R_{g}$ values of ubiquitin in water and in the gas phase. In contrast to Figure 3 (main text) the MD data for this figure were generated without temperature annealing. "Crystal" represents the X-ray structure. Solution: "Piana 2013" refers to native and unfolded MD trajectory segments from ref. $^{74}$, each averaged over $10 \mu \mathrm{s}$; "this work" is for solution runs that started from $\mathrm{U}_{\text {stretched. }}$ Gas Phase: $R_{g}$ values for final structures after stepwise $13+\rightarrow 3+$ proton stripping were calculated for different types of MD runs: "mobile proton", "static proton $\mathrm{A}^{\text {" }}\left(\mathrm{H}^{+}\right.$removal from acidic sites), and "static proton $\mathrm{B}$ " $\left(\mathrm{H}^{+}\right.$removal from basic sites). The last data set is for $13+\rightarrow 3+$ proton stripping in a single step. 